\title{
Paroxysmal Atrial Fibrillation: Insight Into the Intimate Mechanisms of Coagulation
}

\author{
Mariya Negreva $^{\mathrm{a}, \mathrm{d}}$, Ana Zarkova ${ }^{\mathrm{b}}$, Krasimira Prodanova $^{\mathrm{c}}$, Petar Petrov $^{\mathrm{a}}$
}

\begin{abstract}
Background: Atrial fibrillation (AF) is a hypercoagulable state. However, the intimate mechanisms leading to impaired coagulation and the timing of their activation are unclear. The aim of the study was to investigate the factors that initiate the coagulation cascade in the early hours (up to $48 \mathrm{~h}$ ) of clinical manifestation of paroxysmal atrial fibrillation (PAF).
\end{abstract}

Methods: Tissue factor (TF) level, coagulation activity of factor VII (FVIIa), factor XII (FXIIa) and factor XI (FXIa) were measured in plasma of 51 non-anticoagulated patients ( 26 men and 25 women, aged $59.84 \pm 11.42$ years) and 52 controls ( 26 men and 26 women, aged 59.50 \pm 10.53 years) by enzyme-linked immunoassays and kinetic assays.

Results: TF was higher in the PAF group $(268.63 \pm 90.62 \mathrm{pg} / \mathrm{mL}$ vs. $170.21 \pm 66.19 \mathrm{pg} / \mathrm{mL}, \mathrm{P}<0.001)$ as well as FVIIa $(170.82 \pm 59.39 \%$ vs. $95.17 \pm 37.90 \%, \mathrm{P}<0.001)$, FXIIa $(218.31 \pm 84.04 \%$ vs. $148.41 \pm 53.94 \%$, $\mathrm{P}<0.001)$ and FXIa $(178.41 \pm 55.94 \%$ vs. $111.75 \pm 37.33 \%, \mathrm{P}<0.001)$. Regression analysis showed that in the first $6 \mathrm{~h}$ of the disease, increase in time led to increase in FXIIa $(\mathrm{r}=0.25, \mathrm{P}<0.05)$, FXIa $(\mathrm{r}=0.75, \mathrm{P}$ $<0.05)$, TF level $(\mathrm{r}=0.25, \mathrm{P}<0.05)$ and FVIIa $(\mathrm{r}=0.25, \mathrm{P}<0.05)$.

Conclusion: Hemocoagulation changes were observed even $<6 \mathrm{~h}$ after the onset of the disease. They suggest that PAF has an early tendency for hypercoagulability, with the involvement of the intrinsic and extrinsic pathways of coagulation.

Keywords: Atrial fibrillation; Coagulation; Intimate mechanisms

\section{Introduction}

One of the major clinical problems that outlines atrial fibrillation $(\mathrm{AF})$ is the significant risk of cardioembolic events $[1,2]$.

Manuscript submitted November 29, 2019, accepted January 10, 2020

aDepartment of Cardiology, Medical University of Varna, Varna, Bulgaria bNational Specialized Hospital for Active Treatment of Hematologic Diseases, Sofia, Bulgaria

'Faculty of Applied Mathematics and Informatics, Technical University of Sofia, Sofia, Bulgaria

${ }^{\mathrm{d} C}$ Corresponding Author: Mariya Negreva, Department of Cardiology, Medical University of Varna, Varna, Bulgaria. Email: mnegreva@abv.bg

doi: https://doi.org/10.14740/cr972
At their core is the formation of left atrial appendage thrombus $[3,4]$. Undoubtedly, the process is multifactorial and not only associated with a left atrial blood flow disorder and its structural remodeling. Significant changes in a number of hemostasis indicators have also been identified [5, 6]. In this regard, a conducted immunohistochemical study of left atrial appendage in AF patients showed fibrin abundance, significantly exceeding platelet content [7]. This fact greatly clarifies the pathophysiology of AF-related thrombus formation, giving reason to believe that impaired blood coagulation plays a leading role in thromboembolic events in AF.

Over the last two decades, there have been significant changes in the understanding of the blood coagulation process. The classic cascade model, introduced by Macfarlane, Davie and Ratnoff back in 1964, identifies two activation pathways: an extrinsic (initiated by tissue factor (TF)) and an intrinsic pathway (initiated by coagulation factor XII (FXII)), which function separately and are capable of independently inducing the formation of fibrin thrombi [8]. However, clinical observations have shown that this model does not accurately reflect in vivo hemocoagulation. Therefore, a new hypothesis has been proposed, in which a key point is a clear distinction between physiological activation and pathological thrombosis [9]. The two processes are defined as qualitatively different with different regulatory mechanisms. In healthy people, the coagulation cascade is constantly activated externally. Under the initiating action of the TF/FVIIa complex, low levels of activated coagulation factors are generated mainly outside the blood vessels, which impedes the completion of coagulation. However, in conditions of thrombosis, the internal coagulation pathway is activated as a rule. The activated FXII (FXIIa) and FXI (FXIa) factors in in vitro conditions stimulate additional thrombin synthesis, which in turn provides for the propagation and stabilization of thrombi. Therefore, the modern model of hemocoagulation considers the simultaneous activation of both pathways of the coagulation cascade as an integral part of the thrombus formation.

Despite significant developments in the basic understanding of hemocoagulation, there is still uncertainty about the exact pathophysiological mechanisms that initiate the coagulation cascade in thrombotic conditions. After clinical studies have shown normal hemostatic capacity and absence of abnormal bleeding in congenital FXII deficiency, the involvement of FXII in in vivo coagulation remains controversial. In this sense, we can say that the intimate mechanisms that determine the tendency for hypercoagulability in AF remain unclear. There is also no clear answer when they are activated in time. Clini- 
cal evidence indicates that, although lower, thrombotic risk is observed not only in the persistent form of the disease but also in paroxysmal atrial fibrillation (PAF). This determines the scientific and clinical interest in coagulation changes occurring in short episodes of the disease. So far, data on coagulation activity in the early hours of PAF are conflicting. For example, elevated levels of fibrinopeptide A, thrombin-antithrombin III complex, d-dimer and others have been reported in some studies [10-12]. Other studies did not find changes in the fibrin coagulation system in PAF [13-15]. Clarifying these details would undoubtedly lead to refinement of the anticoagulant approach in patients with PAF.

The aim of the study was to investigate the intimate mechanisms that initiate the coagulation cascade in the early hours (up to $48 \mathrm{~h}$ ) of the clinical manifestation of PAF.

\section{Materials and Methods}

\section{Study population}

The subjects of the study were patients with a PAF episode < $48 \mathrm{~h}$ before hospitalization, who clearly indicated its onset as "palpitations". The diagnosis was accepted after verifying it from an electrocardiogram (ECG) record. Of the 338 patients screened, 51 (26 men and 25 women) with a mean age of 59.84 \pm 11.42 years ( 31 - 77 years) were sequentially selected. Two hundred eighty-seven patients were dropped because of exclusion criteria (see below).

A control group of volunteers with no anamnestic or electrocardiographic AF data were formed. For this purpose, 169 outpatients, who had visited their general practitioner (GP) for an annual check-up, were screened. Of these, 52 (26 men and 26 women) were included in the study. Their mean age was $59.50 \pm 10.53$ years (30 - 76 years). The above-mentioned exclusion criteria were applied.

The patients and control group were equalized by gender, age, body mass index (BMI), deleterious habits, comorbidities and their treatment to eliminate their effect on the hemostatic profile.

Excluding criteria were diseases and conditions that themselves alter the hemostatic profile: 1) Cardiovascular diseases: ischemic heart disease, heart failure, high-grade and/or uncontrolled hypertension, moderate or severe acquired valve defects, cardiomyopathy, implanted device for the treatment of rhythm-conduction disorders, inflammatory heart disease, congenital heart diseases; 2) Other diseases: kidney or liver failure, inflammatory and/or infectious diseases, neoplastic and autoimmune diseases, chronic pulmonary insufficiency, endocrine disorders (except for non-insulin dependent, well-controlled diabetes mellitus (DM) type 2); previous thromboembolic incidents, bleeding diathesis, miscarriages (for women); 3) Intake of hormone replacement therapy, contraceptives, oral anticoagulants or antiplatelet drugs, pregnancy, systemic intake of analgesics (incl. nonsteroidal antiinflammatory drugs (NSAIDs)), obesity with BMI > 35; 4) Unsuccessful restoration of sinus rhythm with drugs (propafenone) (for the patient group).

\section{Study design}

The clinical study was conducted at the First Cardiology Clinic of the University Hospital Saint Marina, Varna, Bulgaria after approval by the local ethics committee (Institutional Reviewer Board Approval 9/14.10.2010). The study was carried out in accordance with the Declaration of Helsinki for the period from October 2010 to May 2012 [16].

Peripheral venous blood was collected once in each selected patient immediately after hospitalization prior to initiation of drug treatment. Controls were also examined once.

Four hemocoagulation indicators were examined in each study participant, namely, TF level, coagulation factor VII activity (FVIIa), coagulation factor XII activity (FXIIa) and coagulation factor XI activity (FXIa).

\section{Collection and storage of blood samples}

The indicators were examined in venous blood obtained after peripheral venipuncture (left or right cubital vein). Blood samples were collected in coagulation $3.2 \%$ sodium citrate tubes (VACUETTE, Greiner Bio-One North America, Inc.). Subsequently, they were centrifuged at 2,500 rpm for $15 \mathrm{~min}$, and the resulting plasma was separated and stored according to the manufacturer's requirements for the tests used.

Each indicator was examined twice and the arithmetic mean was taken into account.

\section{Laboratory procedures}

An enzyme-linked immunoassay technique was applied to determine TF concentration in plasma (IMUBIND tissue factor ELISA, BioMedica Diagnostics, USA). The coagulation activity of FVII, FXI and FXII was measured by means of kinetic enzyme tests (factor VII deficient plasma, immunads. Technoclone, Austria; factor XI and XII deficient plasma, native Technoclone, Austria, respectively).

\section{Statistical methods}

Continuous variables were expressed as mean \pm standard deviation (SD) and categorical variables were expressed as percentage of the total group. Two-tailed Student's $t$-test for independent samples was used to compare quantitative variables. Fisher's exact or Pearson's Chi-square tests were used to compare categorical variables and occurrence frequency. Values $\mathrm{P}<0.05$ were adopted for statistically significant. Linear regression analysis was used to analyze the association between the dependent variables TF level, FVIIa, FXIIa and FXIa and the duration of PAF episode. The relationship between hemostatic indicators and the categorical independent variable CHA2DS2-VASc score was examined using analysis of variance (ANOVA). STATISTICA 13.3.0 (StatSoft Inc., USA) software package was used for all statistical analysis. 
Table 1. Clinical Characteristics of the Participants

\begin{tabular}{|c|c|c|c|}
\hline & Patients with PAF & Control group & P values \\
\hline Number of participants & 51 & 52 & 0.89 \\
\hline Mean age (years) & $59.84 \pm 11.42$ & $59.50 \pm 10.53$ & 0.87 \\
\hline \multicolumn{4}{|l|}{ Accompanying diseases } \\
\hline Hypertension & $37(72.54 \%)$ & $34(65.38 \%)$ & 0.44 \\
\hline \multicolumn{4}{|c|}{ Medicaments for hypertension and dyslipidemia } \\
\hline Beta blockers & $19(37.25 \%)$ & $17(32.69 \%)$ & 0.62 \\
\hline ACE inhibitors & $15(29.41 \%)$ & $14(26.92 \%)$ & 0.78 \\
\hline Sartans & $11(21.57 \%)$ & $9(17.31 \%)$ & 0.58 \\
\hline Alcohol intake & $7(13.72 \%)$ & $6(11.53 \%)$ & 0.74 \\
\hline BMI $\left(\mathrm{kg} / \mathrm{m}^{2}\right)$ & $23.85 \pm 3.28$ & $24.95 \pm 3.24$ & 0.09 \\
\hline CHA2DS2-VASc score & & No score & \\
\hline Number of patients with score $<2$ & 25 & & \\
\hline Number of patients with score $\geq 2$ & 26 & & \\
\hline
\end{tabular}

CHA2DS2-VASc score - congestive heart failure, hypertension, age $\geq 75$ years, diabetes mellitus, stroke/transient ischemic attack/thromboembolism, vascular disease (prior myocardial infarction, peripheral vascular disease, or aortic atherosclerosis), age (65 - 74 years) and sex category (female). According to CHA2DS2-VASc score, patients were divided into low-risk (score <2) and high-risk (score $\geq 2$ ) for the emergence of embologenic risk according to the recommendations of the European Society of Cardiology [17]. PAF: paroxysmal atrial fibrillation; ACE: angiotensin converting enzyme; BMI: body mass index.

\section{Results}

\section{Participants characteristics}

In terms of number, mean age and sex structure, the patient group was comparable to the control $(\mathrm{P}>0.05)$ (Table 1) [17]. In terms of comorbidities, dyslipidemia and treatment (up to hospitalization), as well as frequency of deleterious habits and BMI, the patient group with PAF was statistically identical to control group $(\mathrm{P}>0.05)$ (Table 1). Data from the transthoracic echocardiographic study also showed no statistically significant differences in the studied parameters between patients and controls $(\mathrm{P}>0.05)$ (Table 2$)$.

The statistical analysis of the time of manifestation of $\mathrm{AF}$ prior to hospitalization showed that all 51 patients were hospitalized between the second and 24th hour after the onset of arrhythmia, most often during the fifth hour. No patient was admitted to the ward after the 24th hour, and the mean duration of arrhythmic episodes prior to hospitalization was $8.14 \pm 5.40 \mathrm{~h}$.

Table 2. Echocardiographic Evaluation of the Participants

\begin{tabular}{llll}
\hline Echocardiographic indicators & Patients with PAF & Control group & P values \\
\hline LVEDD $(\mathrm{mm})$ & $52.57 \pm 5.43$ & $52.29 \pm 5.44$ & 0.73 \\
LVESD $(\mathrm{mm})$ & $34.43 \pm 5.34$ & $34.73 \pm 4.99$ & 0.69 \\
EF $(\%)$ & $62.98 \pm 5.97$ & $61.54 \pm 5.49$ & 0.12 \\
IVS $(\mathrm{mm})$ & $10.37 \pm 3.42$ & $9.92 \pm 3.68$ & 0.20 \\
PW (mm) & $10.24 \pm 3.27$ & $9.73 \pm 3.82$ & 0.16 \\
LA volume $\left(\mathrm{mL} / \mathrm{m}^{2}\right)$ & $22.81 \pm 4.80$ & $23.82 \pm 5.00$ & 0.13 \\
RVEDD $(\mathrm{mm})$ & $30.54 \pm 8.98$ & $29.17 \pm 8.51$ & 0.18 \\
\hline
\end{tabular}

PAF: paroxysmal atrial fibrillation; LVEDD: left ventricular end-diastolic dimension; LVESD: left ventricular end-systolic dimension; EF: ejection fraction; IVS: interventricular septum thickness; PW: posterior wall; LA: left atrial; RVEDD: right ventricular end-diastolic dimension. 


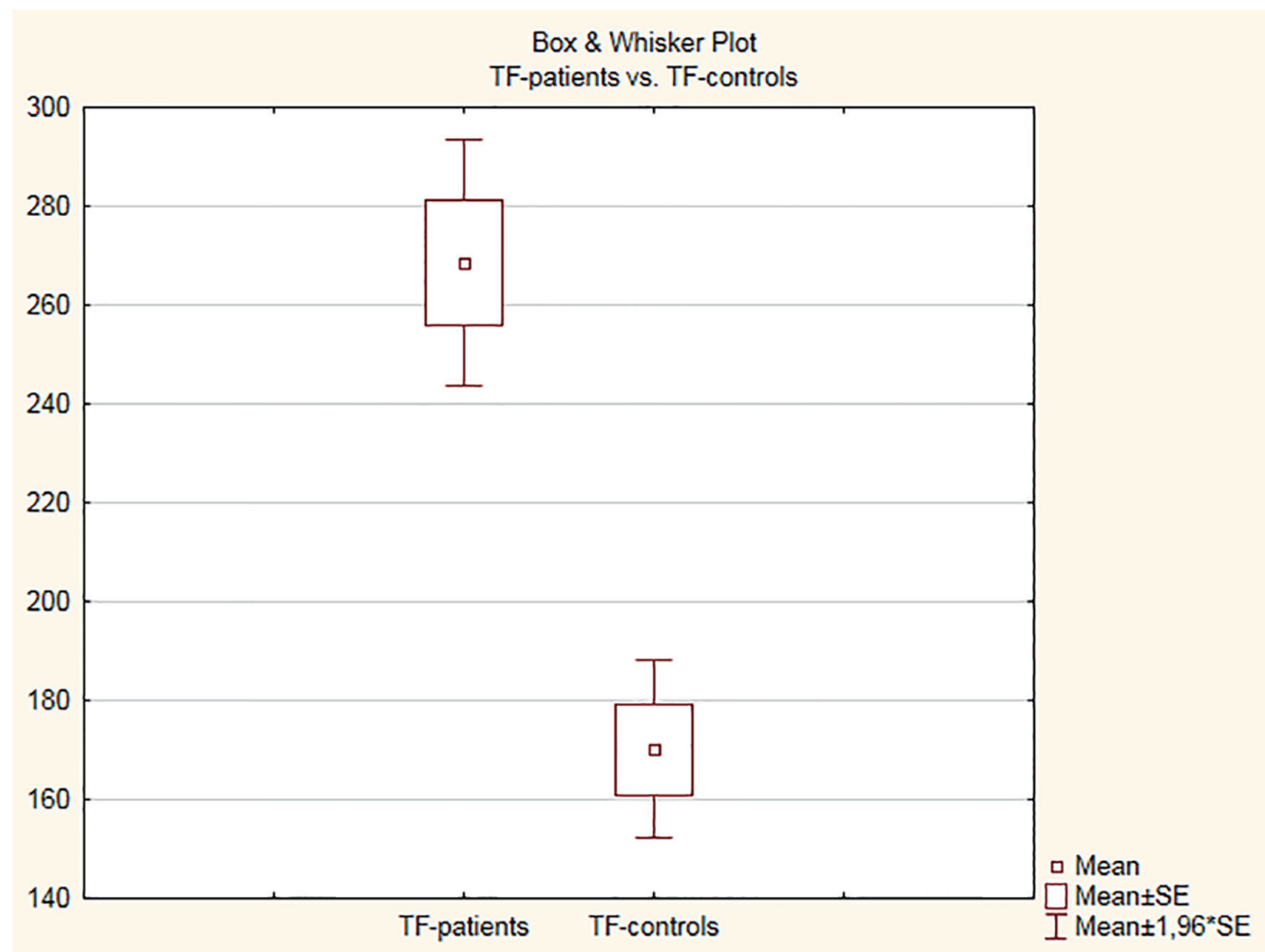

Figure 1. Tissue factor levels in plasma in the control and patient groups.

\section{Coagulation markers}

Plasma level of TF was significantly higher in PAF group (268.63 $\pm 90.62 \mathrm{pg} / \mathrm{mL}$ vs. $170.21 \pm 66.19 \mathrm{pg} / \mathrm{mL}, \mathrm{P}<0.001$; Fig. 1). Higher activity of FVIIa $(170.82 \pm 59.39 \%$ vs. $95.17 \pm 37.90 \%$, P $<0.001$; Fig. 2), FXIIa (218.31 $\pm 84.04 \%$ vs. $148.41 \pm 53.94 \%$, P $<0.001$; Fig. 3) and FXIa (178.41 $\pm 55.94 \%$ vs. $111.75 \pm 37.33 \%$, $\mathrm{P}<0.001$; Fig. 4) and was also measured in patients with AF.

A significant correlation was found between FXIIa and time spent in PAF, an increase in time in PAF led to increase in the activity of the coagulation factor $\left(\mathrm{r}=0.78\right.$, adjusted $\mathrm{R}^{2}$ $=0.61, \mathrm{P}<0.001$, Fig. 5). No linear dependence was found between TF levels $\left(\mathrm{r}=0.16\right.$, adjusted $\left.\mathrm{R}^{2}=0.01 ; \mathrm{P}>0.05\right)$, FVIIa $\left(r=-0.20\right.$, adjusted $\left.\mathrm{R}^{2}=0.02, \mathrm{P}>0.05\right)$, FXIa $(\mathrm{r}=0.20$, adjusted $\mathrm{R}^{2}=0.02, \mathrm{P}>0.05$ ) and the time spent in PAF.

Additionally, the patient group was divided into two groups according to onset of the arrhythmia: the first group included patients with arrhythmia duration $\leq 6 \mathrm{~h}$, and the second group included patients with arrhythmia duration between 7 and $24 \mathrm{~h}$. Regression analysis showed that FXIIa $(\mathrm{r}=0.25$, Fig. 6), FXIa ( $\mathrm{r}=0.75$, Fig. 7), TF level $(\mathrm{r}=0.25$, Fig. 8$)$ and FVIIa $(r=0.25$; Fig. 9) increased in the first group of patients (PAF duration $\leq 6 \mathrm{~h}$ ) with increase in the time spent in PAF. In the second group of patients, FXIIa showed a correlation with PAF duration $(r=0.39)$. Such correlation was not found for FXIa $(r=-0.08)$ and TF $(r=0.005)$. FVIIa showed a negative correlation $(\mathrm{r}=-0.25)$.

ANOVA did not find any statistically significant difference between patients with low (CHA2DS2-VASc score <
2) and high embologenic risk (CHA2DS2-VASc score $\geq 2$ ) with respect to all four studied indicators, namely TF levels $(260.55 \pm 91.42 \mathrm{pg} / \mathrm{mL}$ vs. $276.29 \pm 90.96 \mathrm{pg} / \mathrm{mL}, \mathrm{P}>0.05)$, FVIIa $(181.57 \pm 57.72 \%$ vs. $160.47 \pm 60.24 \%, \mathrm{P}>0.05)$, FXIIa $(212.41 \pm 79.92 \%$ vs. $224.01 \pm 89.03 \%, \mathrm{P}>0.05)$ and FXIa $(178.31 \pm 55.62 \%$ vs. $178.53 \pm 57.34 \%, \mathrm{P}>0.05)$.

\section{Discussion}

Thromboembolism is generally the most common cause of morbidity and mortality worldwide [18]. Its pathophysiology has its specific characteristics in various diseases: platelet activation plays a key role in arterial atherosclerotic incidents, and stasis and hypercoagulability are crucial for the development of deep vein thrombosis and AF [19]. Regardless of the differences, a common principle in thrombus formation is the activation of the coagulation cascade. Therefore, hemocoagulation and its intimate mechanisms are of scientific and clinical interest both in the general context of thrombus formation and in individual prothrombotic cases.

Elevated levels of some coagulation activity indicators such as d-dimer, fibrinogen, antithrombin III (ATIII), thrombin-antithrombin III complex, prothrombin fragments $1+2$ $(\mathrm{PF} 1+2)$ and others have been observed in AF patients [20]. This gives us a general reason to assumption that the disease is associated with activated hemocoagulation. However, the presented studies so far, have examined single indicators, which do not allow revealing the nature of the identified changes. 
Box \& Whisker Plot

FVII-patients vs. FVII-controls

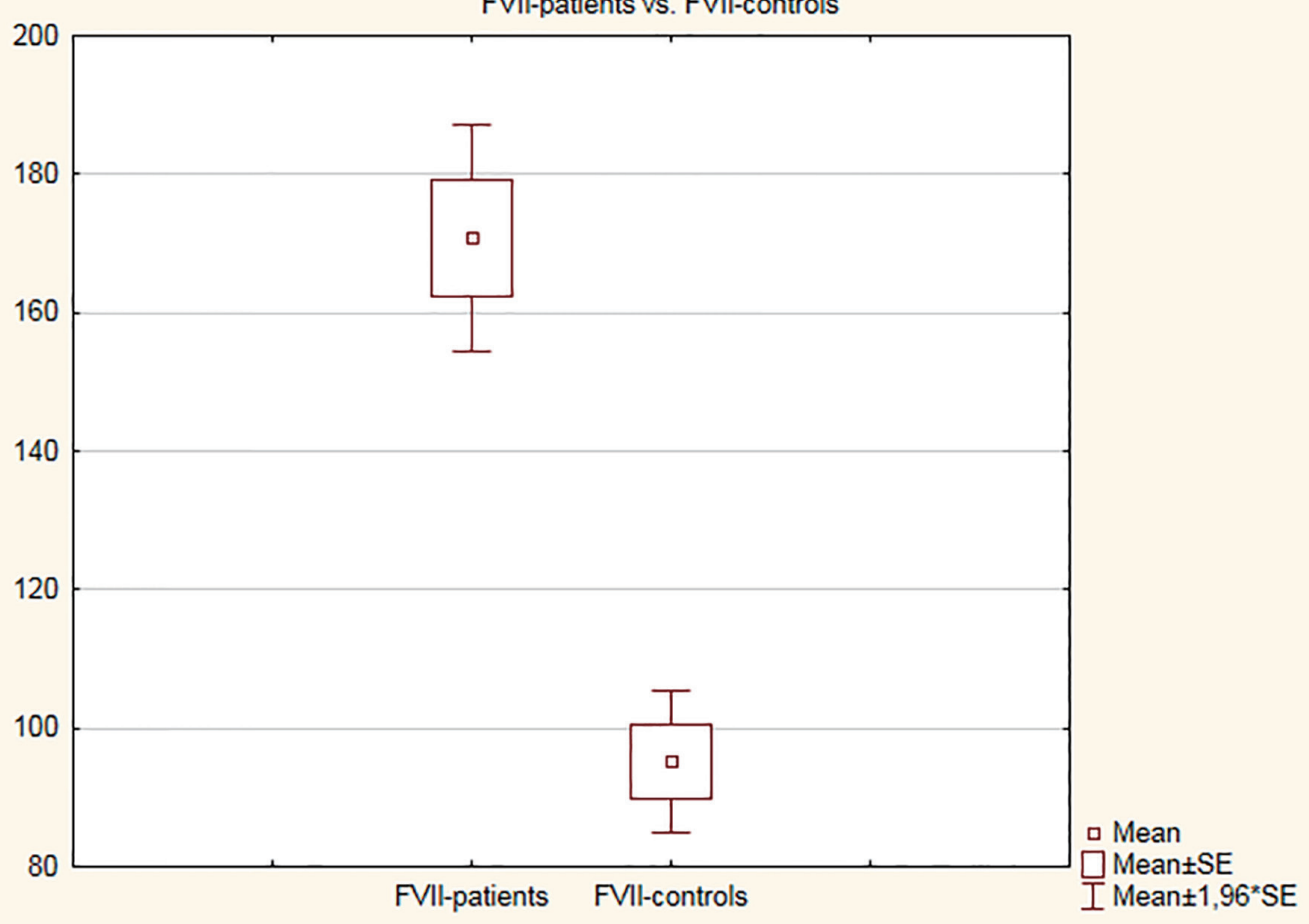

Figure 2. FVII coagulation activity in the control and patient groups.

Box \& Whisker Plot

FXII-patients vs. FXII-controls

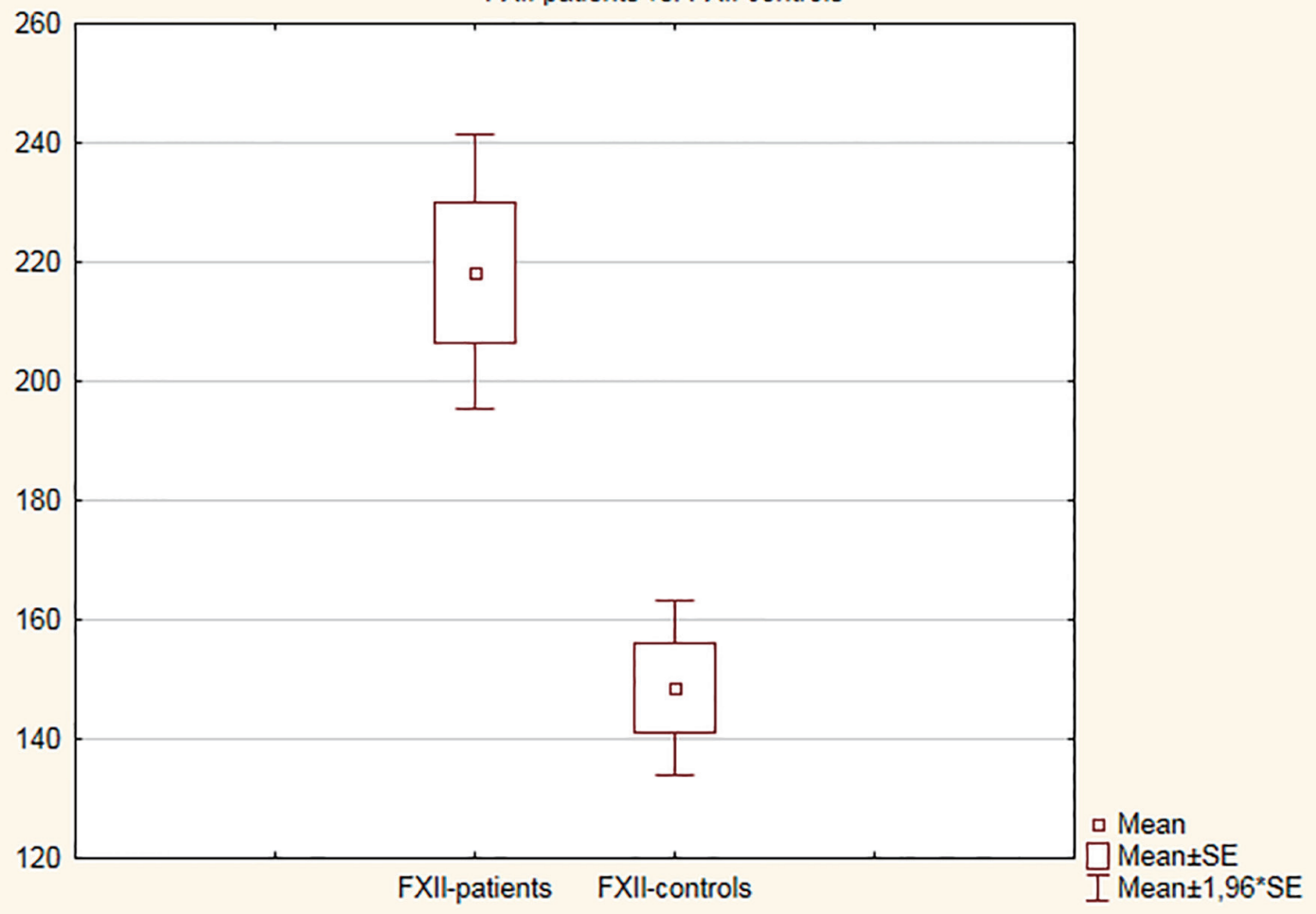

Figure 3. FXII coagulation activity in the control and patient groups. 
Box \& Whisker Plot FXI-patients vs. FXI-controls

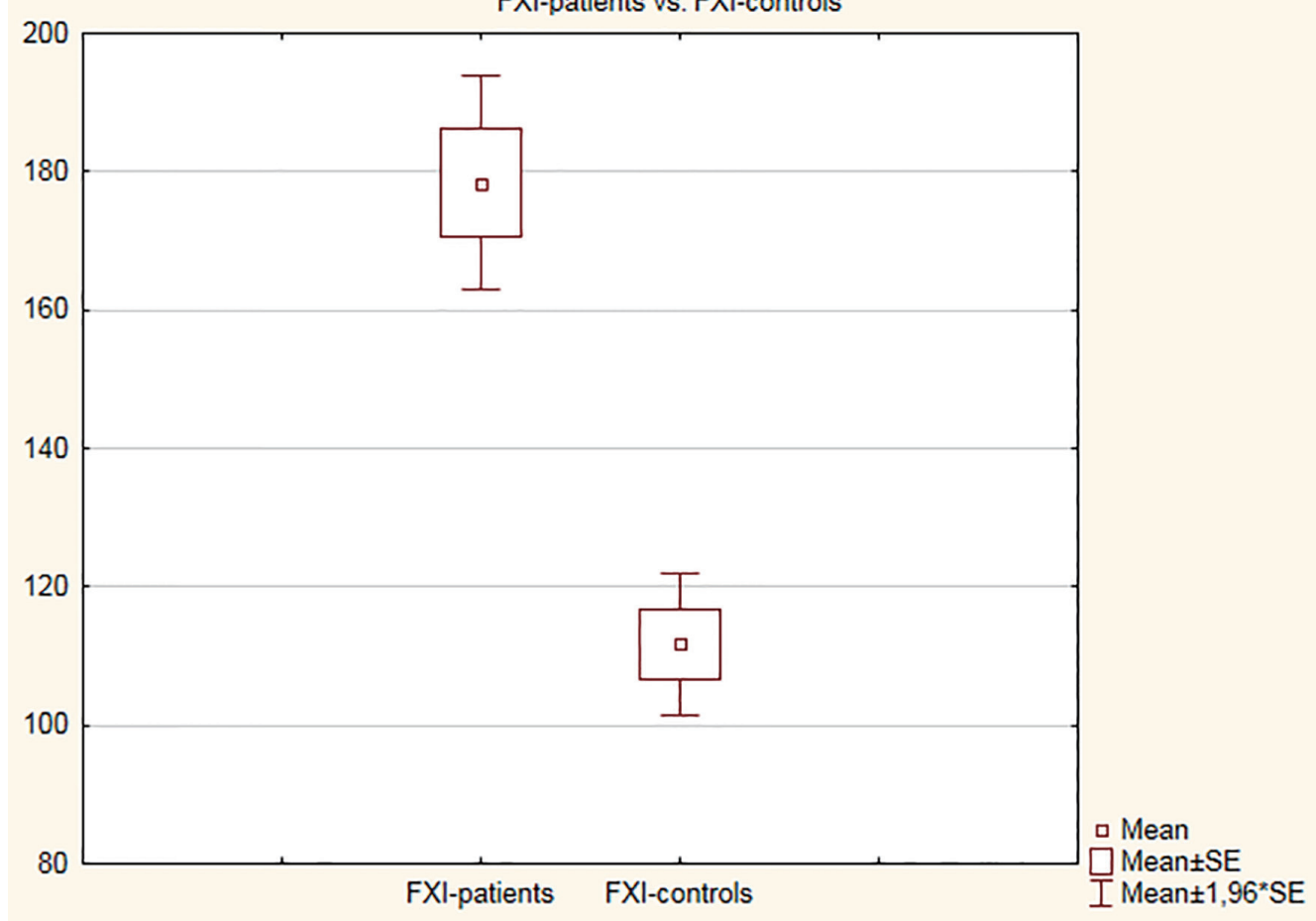

Figure 4. FXI coagulation activity in the control and patient groups.

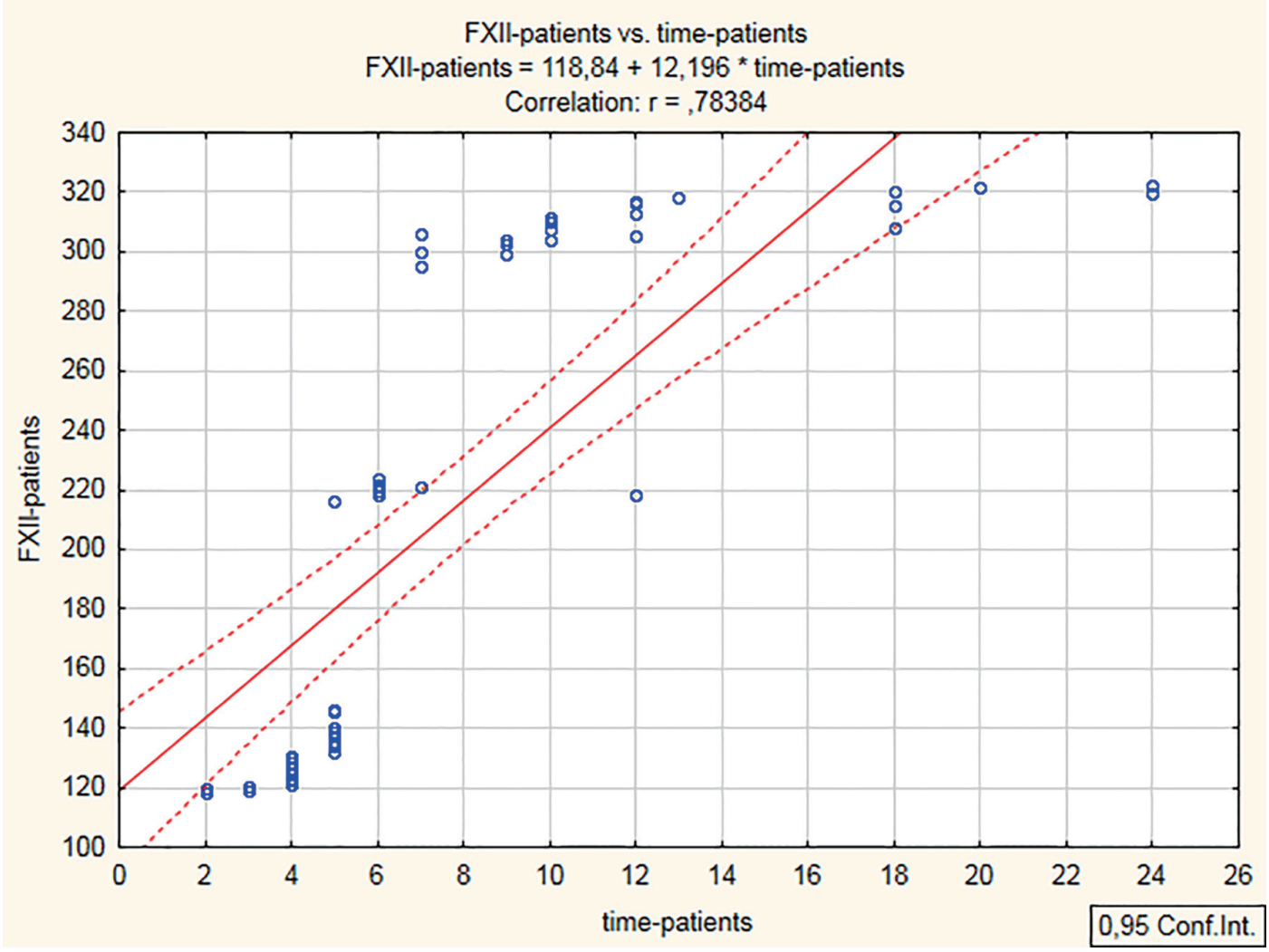

Figure 5. Correlation between XII activity and time spent in PAF. PAF: paroxysmal atrial fibrillation. 


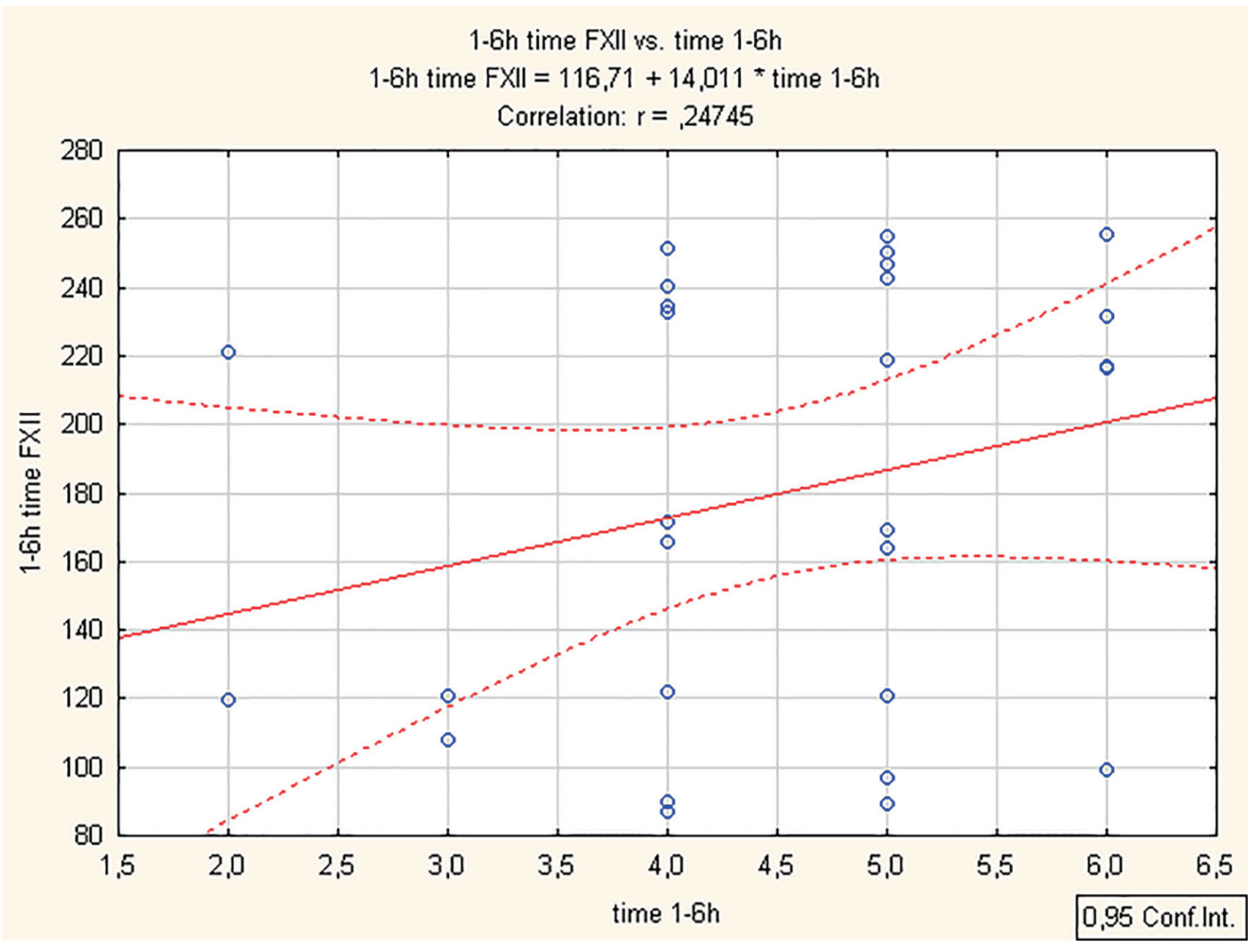

Figure 6. Changes in FXIla during the first $6 \mathrm{~h}$ of the clinical manifestation of PAF. PAF: paroxysmal atrial fibrillation.

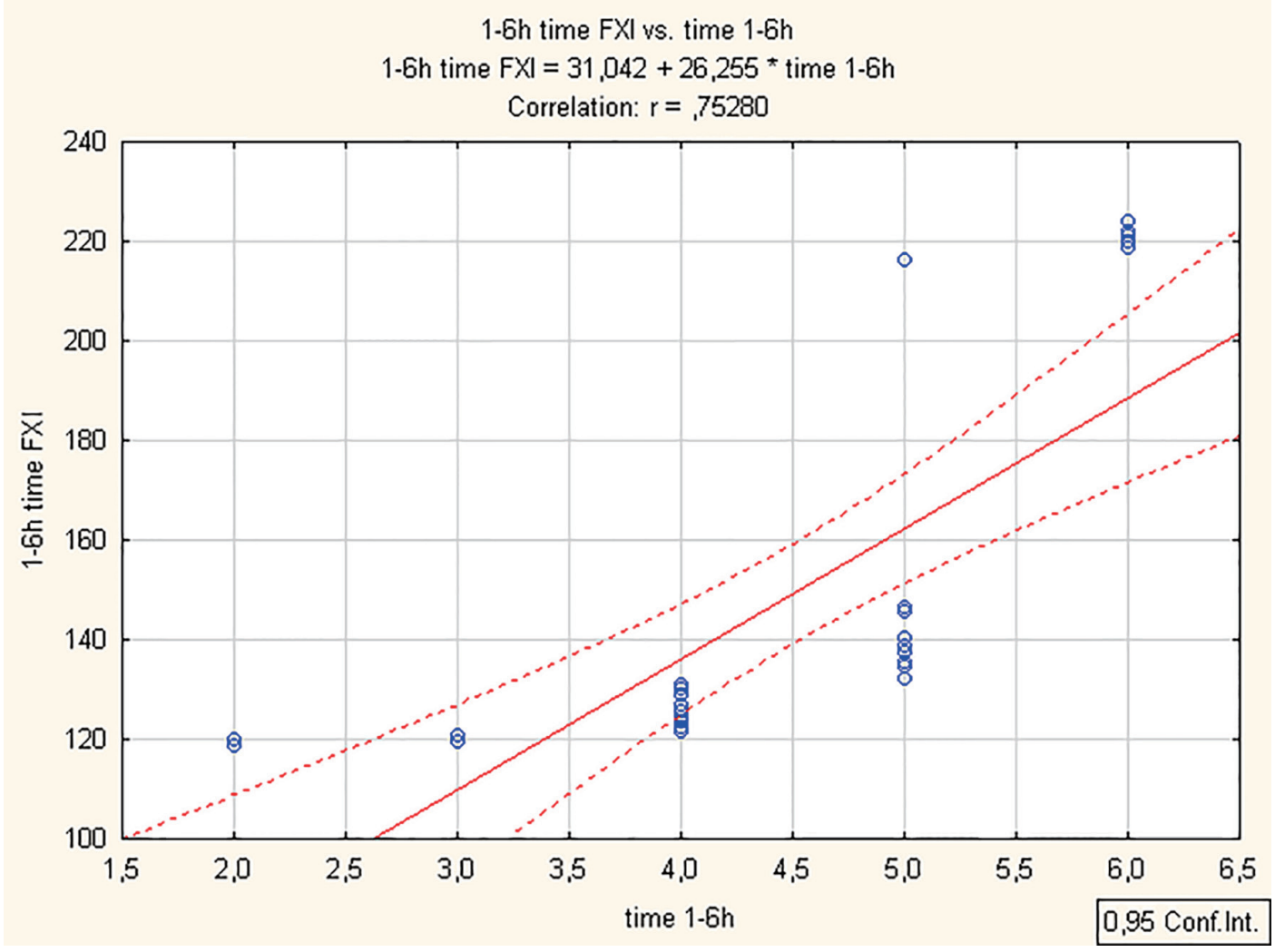

Figure 7. Changes in FXla during the first $6 \mathrm{~h}$ of the clinical manifestation of PAF. PAF: paroxysmal atrial fibrillation. 


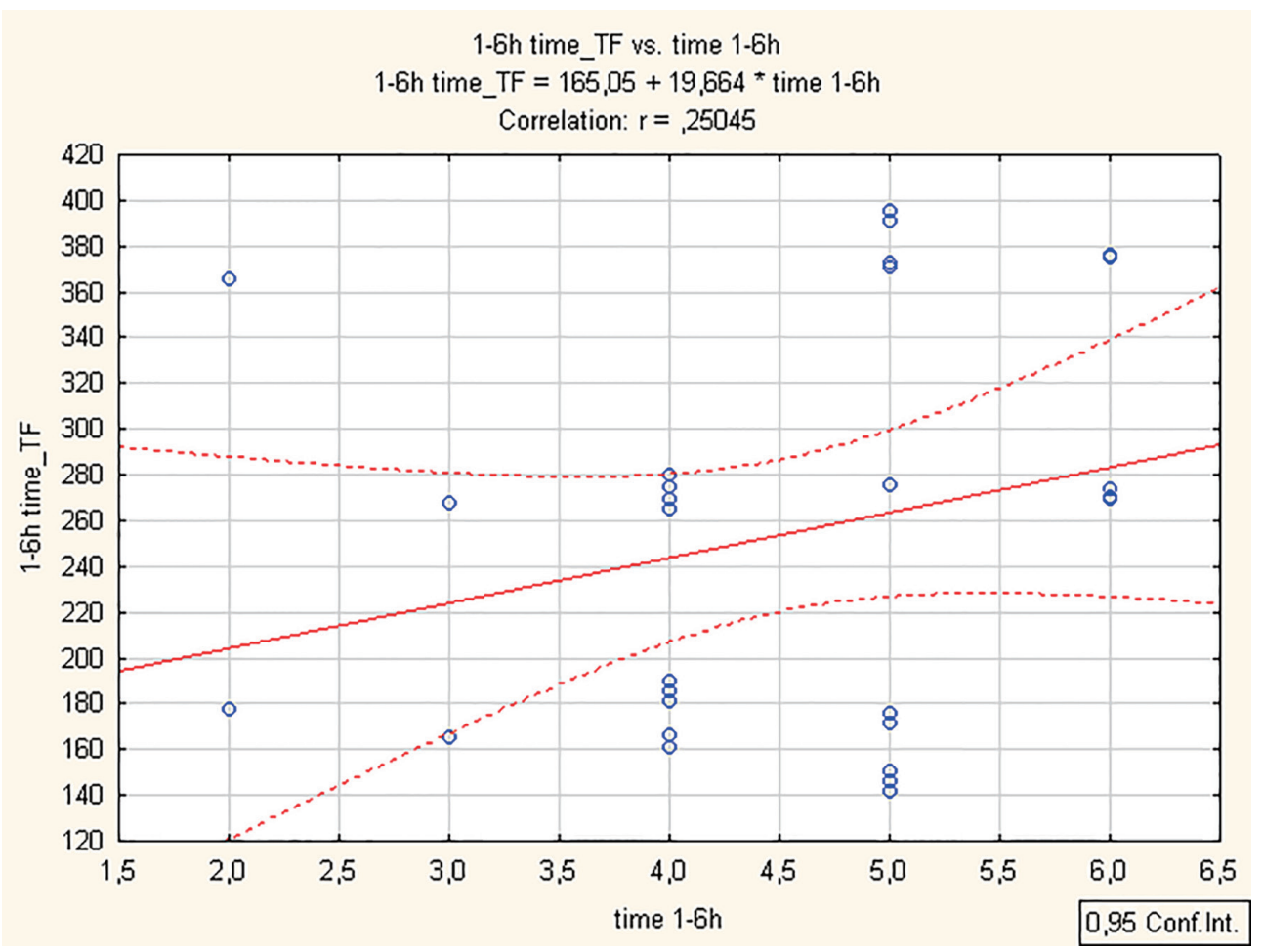

Figure 8. Changes in TF plasma level during the first $6 \mathrm{~h}$ of the clinical manifestation of PAF. TF: tissue factor; PAF: paroxysmal atrial fibrillation.

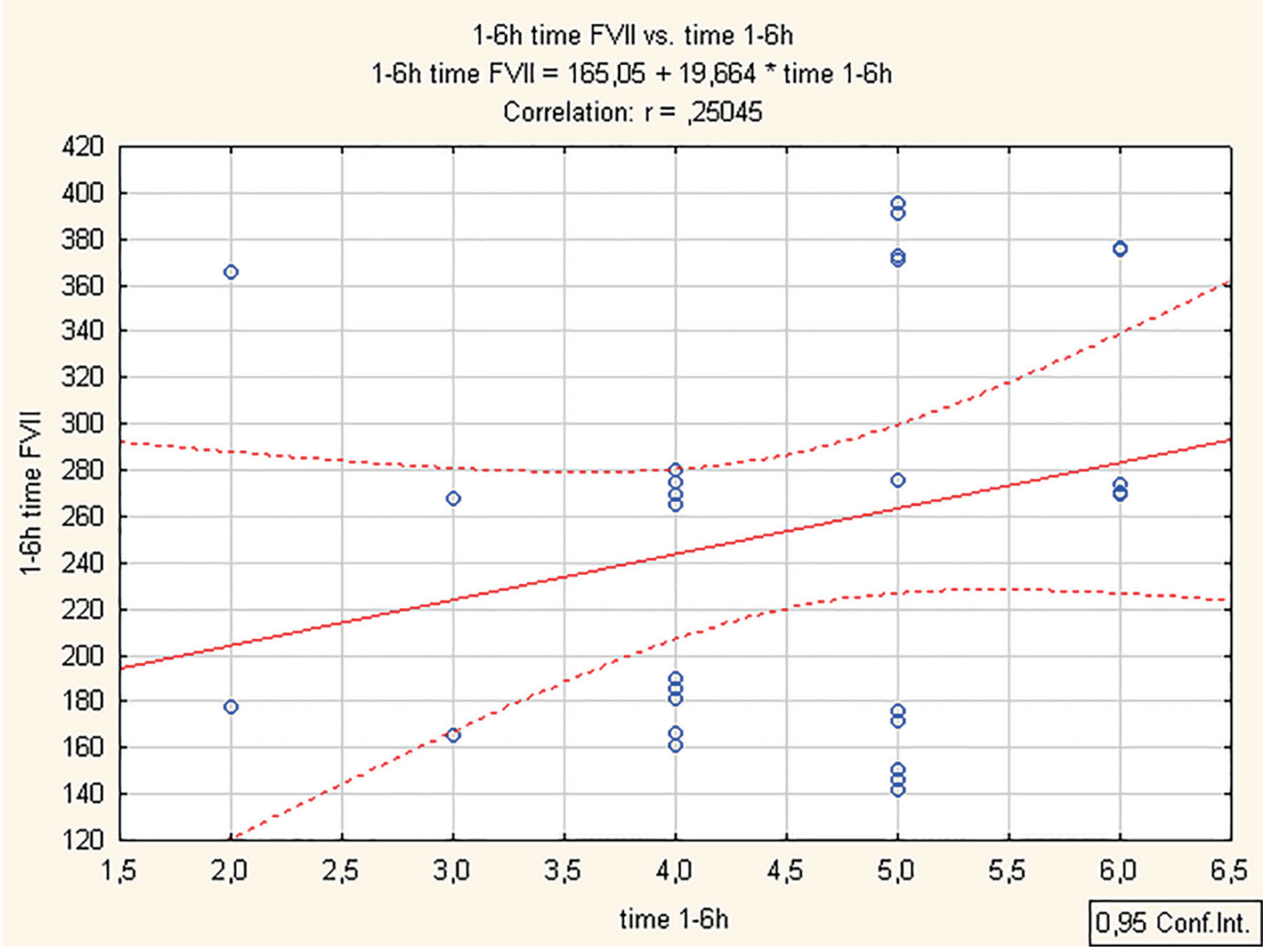

Figure 9. Changes in FVIla during the first $6 \mathrm{~h}$ of the clinical manifestation of PAF. PAF: paroxysmal atrial fibrillation. 
Patient groups are predominantly with persistent or permanent AF, which does not allow to time the changes. Studies on PAF are few and controversial. High levels of fibrinogen were measured as early as the early hours (up to $48 \mathrm{~h}$ ) of the disease $[14,15,21]$. Increased levels of d-dimer have also been identified as an indirect marker of enhanced coagulation, as well as of the thrombin-antithrombin complex [22-24]. Other clinical studies have found no changes in hemocoagulation in PAF $[13,25]$. This further complicates the analysis and generalization of currently available data on coagulation in PAF.

According to current concepts, TF plays a key role in the in vivo activation of coagulation. It is the universal initiating factor of the coagulation cascade both under physiological and thrombotic conditions. In its nature, it is a transmembrane receptor. The binding to its sole ligand FVII/FVIIa initiates coagulation by activating both FX and FIX - a central molecule in the intrinsic coagulation pathway [26]. Moreover, the TF/ FVIIa-dependent initial activation of FIX is considered to be of even greater significance than the FXIa-mediated one [27]. This is evidence of the close relationship between the two pathways, their functional unity and the determining role of TF and FVIIa in the activation of hemocoagulation. As can be seen from our results (Figs. 1 and 2), plasma TF levels and coagulation activity of FVIIa were significantly higher in PAF patients than the controls $(\mathrm{P}<0.001)$. It is well known that TF is not only expressed on the endothelial surface and its damage is not the only source. TF can enter the bloodstream with fragmented membrane particles of various cell types such as leukocytes and platelets, as well as in activation of endothelial cells and monocytes $[28,29]$. Possibly the high levels of TF in the study patient group are of multicomponent origin, given the variety of mechanisms that can provoke its contact with blood components. High TF levels are followed by significant activation of FVII, which was also observed in our study. The changes we have detected in TF and FVIIa suggest that the extrinsic pathway of the coagulation cascade is significantly activated in the first $24 \mathrm{~h}$ of the clinical manifestation of PAF. This in turn determines the enhanced activation of the initiation phase of the coagulation process.

FXII and FXI play an important role in the next stages of coagulation, namely propagation and ultimate stabilization of thrombi [30]. This gave reason for our clinical interest in the activity of FXII and FXI. Unidirectional deviations were detected (Figs. 3 and 4; $\mathrm{P}<0.001$ ). The activity of both indicators in the patient group was significantly higher than that of the controls, which is evidence of activation of the intrinsic pathway of the coagulation cascade in the patient population studied by us.

Thromboembolic risk in AF and, respectively, the need for anticoagulant treatment, is determined based on assessment scales, and the most commonly used is the CHA2DS2-VASc score, which includes congestive heart failure, hypertension, age, DM, stroke/transient ischemic attack/thromboembolism, vascular disease (prior myocardial infarction, peripheral vascular disease, or aortic atherosclerosis) and sex category (female) $[17,31]$. These scales include factors that are considered embologenic, i.e. determining increased coagulation activity on their own. AF is not present in any of the assessment scales used. However, our ANOVA showed that there were no significant differences in TF levels, FVIIa, FXIIa and FXIa be- tween patients with low (CHA2DS2-VASc score $<2$ ) and high (CHA2DS2-VASc score $\geq 2$ ) embologenic risk $(\mathrm{P}>0.05)$. In the examined PAF population, coagulation activity was increased regardless of the embologenic risk. This led us to believe that the manifestation of PAF alone could not be ignored as a factor that independently affects coagulation activity and, respectively, embolic risk. We believe that our results provide a good basis for further studies to include the disease itself in the assessment of the embologenic risk associated with it and to refine the possibilities for doing so. On the other hand, regression analysis showed that arrhythmia duration was a predictor of FXIIa values $(\mathrm{r}=0.78, \mathrm{P}<0.05)$. As duration increases, FXIIa increases as well ( $\mathrm{r}=0.78, \mathrm{P}<0.001$; Fig. 5). Adjusted correlation coefficient $\mathrm{R}^{2}$ showed that a significant part of changes in FXIIa could be explained by changes in duration (adjusted $\mathrm{R}^{2}=0.61$ ). The resulting scatter curves showed that most of the data represented coagulation activity by the sixth hour of the onset of the disease (28 patients were admitted with a PAF episode duration $\leq 6 \mathrm{~h}$ ). In this regard, in order to better characterize the regression lines, we divided the patients into two groups according to the episode duration: with a PAF duration $\leq 6 \mathrm{~h}$ and $>6 \mathrm{~h}$. The results showed that in the first 6 $\mathrm{h}$ of the disease, the values of all four indicators increased with duration, suggesting a tendency towards hypercoagulability to be formed still in these early hours.

The "48-h rule" is well established in clinical practice, according to which early cardioversion (up to $48 \mathrm{~h}$ of arrhythmia) is safe with a low thromboembolic risk ranging between $0.3 \%$ and $1.1 \%$ [32]. That is why early IV anticoagulant therapy is currently recommended. The changes we established give us reason to believe that it should be obligatory and arising not from the general embologenic risk but from the very presence of the rhythmic disturbance.

\section{Conclusion}

Changes in hemocoagulation were observed even $<6 \mathrm{~h}$ after the onset of the disease. They suggest an early tendency for hypercoagulability in PAF, with the involvement of the intrinsic and extrinsic pathway.

\section{Limitations of the study}

The indicators were examined once, immediately after hospitalization, which was predetermined by the design of the study. As can be seen from the presented patient characteristics, some patients have a CHA2DS2-VASc score $\geq 2$, which requires anticoagulant treatment in the diagnosis of the disease. The study of coagulation factors against the background of anticoagulation treatment would compromise the reliability of the obtained results.

\section{Acknowledgments}

The third author Krasimira Prodanova is totally supported by 
project DN12/11/20.dec.2017 of the Ministry of Education and Science of Bulgaria for statistical analysis of data.

\section{Financial Disclosure}

The study was not supported by any agency.

\section{Conflict of Interest}

The authors have no conflict of interest to declare.

\section{Informed Consent}

Written informed consent was obtained before study.

\section{Author Contributions}

MN and PP screened and selected the participants. AZ examined the blood samples. KP helped for the statistical analysis. $\mathrm{MN}$ and $\mathrm{AZ}$ analyzed the results. MN wrote the first version of the manuscript. All authors had contribution to the final (sent) version of the manuscript.

\section{References}

1. Kirchhof P, Auricchio A, Bax J, Crijns H, Camm J, Diener $\mathrm{HC}$, Goette A, et al. Outcome parameters for trials in atrial fibrillation: executive summary. Eur Heart J. 2007;28(22):2803-2817.

2. Bekwelem W, Connolly SJ, Halperin JL, Adabag S, Duval S, Chrolavicius S, Pogue J, et al. Extracranial systemic embolic events in patients with nonvalvular atrial fibrillation: incidence, risk factors, and outcomes. Circulation. 2015;132(9):796-803.

3. Manning WJ, Silverman DI, Waksmonski CA, Oettgen P, Douglas PS. Prevalence of residual left atrial thrombi among patients with acute thromboembolism and newly recognized atrial fibrillation. Arch Intern Med. 1995;155(20):2193-2198.

4. Klein AL, Grimm RA, Murray RD, Apperson-Hansen C, Asinger RW, Black IW, Davidoff R, et al. Use of transesophageal echocardiography to guide cardioversion in patients with atrial fibrillation. N Engl J Med. 2001;344(19):1411-1420.

5. Watson T, Shantsila E, Lip GY. Mechanisms of thrombogenesis in atrial fibrillation: Virchow's triad revisited. Lancet. 2009;373(9658):155-166.

6. Lip GYH, Velu S. Haemostasis and thrombosis: focus on atrial fibrillation. In: Lanzer P. (eds) PanVascular Medicine. Springer, Berlin, Heidelberg. 2014

7. Wysokinski WE, Owen WG, Fass DN, Patrzalek DD, Murphy L, McBane RD, 2nd. Atrial fibrillation and thrombosis: immunohistochemical differences between in situ and embolized thrombi. J Thromb Haemost. 2004;2(9):1637-1644.

8. Davie EW, Ratnoff OD. Waterfall Sequence for Intrinsic Blood Clotting. Science. 1964;145(3638):1310-1312.

9. Vojacek JF. Should we replace the terms intrinsic and extrinsic coagulation pathways with tissue factor pathway? Clin Appl Thromb Hemost. 2017;23(8):922-927.

10. Giansante C, Fiotti N, Miccio M, Altamura N, Salvi R, Guarnieri G. Coagulation indicators in patients with paroxysmal atrial fibrillation: effects of electric and pharmacologic cardioversion. Am Heart J. 2000;140(3):423-429.

11. Hatzinikolaou-Kotsakou E, Kartasis Z, Tziakas D, Stakos D, Hotidis A, Chalikias A, Bourikas G, et al. Clotting state after cardioversion of atrial fibrillation: a haemostasis index could detect the relationship with the arrhythmia duration. Thrombosis J. 2005;3(2):1-6.

12. Motoki H, Tomita T, Aizawa K, Kasai H, Izawa A, Kumazaki S, Tsutsui $\mathrm{H}$, et al. Coagulation activity is increased in the left atria of patients with paroxysmal atrial fibrillation during the non-paroxysmal period. Comparison with chronic atrial fibrillation. Circ J. 2009;73(8):1403-1407.

13. Sohara H, Miyahara K. Effect of atrial fibrillation on the fibrino-coagulation system - study in patients with paroxysmal atrial fibrillation. Jpn Circ J. 1994;58(11):821826.

14. Kamath S, Blann AD, Chin BS, Lip GY. Platelet activation, haemorheology and thrombogenesis in acute atrial fibrillation: a comparison with permanent atrial fibrillation. Heart. 2003;89(9):1093-1095.

15. Li-Saw-Hee FL, Blann AD, Gurney D, Lip GY. Plasma von Willebrand factor, fibrinogen and soluble P-selectin levels in paroxysmal, persistent and permanent atrial fibrillation. Effects of cardioversion and return of left atrial function. Eur Heart J. 2001;22(18):1741-1747.

16. World Medical Association (WMA). Declaration of Helsinki. Ethical Principles for Medical Research Involving Human Subjects. Jahrbuch für Wissenschaft und Ethik. 2010;14(1):233-238

17. Kirchhof P, Benussi S, Kotecha D, Ahlsson A, Atar D, Casadei B, Castella M, et al. 2016 ESC Guidelines for the management of atrial fibrillation developed in collaboration with EACTS. Eur J Cardiothorac Surg. 2016;50(5):e1-e88.

18. Raskob GE, Angchaisuksiri P, Blanco AN, Buller H, Gallus A, Hunt BJ, Hylek EM, et al. Thrombosis: a major contributor to global disease burden. Arterioscler Thromb Vasc Biol. 2014;34(11):2363-2371.

19. Mackman N. Triggers, targets and treatments for thrombosis. Nature. 2008;451(7181):914-918.

20. Wu N, Tong S, Xiang Y, Wu L, Xu B, Zhang Y, Ma X, et al. Association of hemostatic markers with atrial fibrillation: a meta-analysis and meta-regression. PLoS One. 2015;10(4):e0124716.

21. Fu R, Wu S, Wu P, Qiu J. A study of blood soluble Pselectin, fibrinogen, and von Willebrand factor levels in idiopathic and lone atrial fibrillation. Europace. 2011;13(1):31-36.

22. Sohara H, Amitani S, Kurose M, Miyahara K. Atrial fi- 
brillation activates platelets and coagulation in a timedependent manner: a study in patients with paroxysmal atrial fibrillation. J Am Coll Cardiol. 1997;29(1):106-112.

23. Marin F, Roldan V, Climent VE, Ibanez A, Garcia A, Marco P, Sogorb F, et al. Plasma von Willebrand factor, soluble thrombomodulin, and fibrin D-dimer concentrations in acute onset non-rheumatic atrial fibrillation. Heart. 2004;90(10):1162-1166.

24. Hou J, Liang Y, Gai X, Zhang H, Yang X, Lan X, Zheng $\mathrm{W}$, et al. The impact of acute atrial fibrillation on the prothrombotic state in patients with essential hypertension. Clin Biochem. 2010;43(15):1212-1215.

25. Kamath S, Chin BS, Blann AD, Lip GY. A study of platelet activation in paroxysmal, persistent and permanent atrial fibrillation. Blood Coagul Fibrinolysis. 2002;13(7):627636.

26. Rao LV, Pendurthi UR. Tissue factor-factor VIIa signaling. Arterioscler Thromb Vasc Biol. 2005;25(1):47-56.
27. Colman RW. Are hemostasis and thrombosis two sides of the same coin? J Exp Med. 2006;203(3):493-495.

28. Rao LV, Kothari H, Pendurthi UR. Tissue factor encryption and decryption: facts and controversies. Thromb Res. 2012;129(Suppl 2):S13-17.

29. Ferreira CN, de Oliveira SM, Maria DL, Carvalho DM. A cell-based model of coagulation and its implications. Rev Bras Hematol Hemoter. 2010;32(5):416-421.

30. Gailani D, Renne T. The intrinsic pathway of coagulation: a target for treating thromboembolic disease? J Thromb Haemost. 2007;5(6):1106-1112.

31. Lip GYH, Banerjee A, Boriani G, Chiang CE, Fargo R, Freedman B, Lane DA, et al. Antithrombotic therapy for atrial fibrillation: CHEST guideline and expert panel report. Chest. 2018;154(5):1121-1201.

32. Rankin AJ, Rankin SH. Cardioverting acute atrial fibrillation and the risk of thromboembolism: not all patients are created equal. Clin Med (Lond). 2017;17(5):419-423. 